\title{
Safety and efficacy of nivolumab for metastatic renal cell carcinoma: real-world results from an expanded access programme
}

\section{Ugo De Giorgi ${ }^{1}$ (D), Giacomo Cartenì ${ }^{2}$, Diana Giannarelli ${ }^{3}$, Umberto Basso ${ }^{4}$, Luca Galli ${ }^{5}$, Enrico Cortesi ${ }^{6}$, Claudia Caserta ${ }^{7}$, Sandro Pignata ${ }^{8}$, Roberto Sabbatini ${ }^{9}$, Alessandra Bearz $^{10}$, Sebastiano Buti ${ }^{11}$, Giovanni Lo Re ${ }^{12}$, Alfredo Berruti ${ }^{13}$, Sergio Bracarda ${ }^{14}$, Francesco Cognetti ${ }^{15}$, Francesca Rastelli ${ }^{16}$, Giuseppe Fornarini ${ }^{17}$, Camillo Porta ${ }^{18,19}$, Daniele Turci ${ }^{20}$, Cora N. Sternberg ${ }^{21}$ (D) and Giuseppe Procopio ${ }^{22}$, on behalf of the Italian Nivolumab Renal Cell Cancer Early Access Program Group ${ }^{a}$}

\footnotetext{
${ }^{7}$ Department of Medical Oncology, Istituto Scientifico Romagnolo per lo Studio e la Cura dei Tumori (IRST) - IRCCS, Meldola, ${ }^{2}$ Department of Medical Oncology, Azienda Ospedaliero-Universitaria "A. Cardarelli", Napoli, ${ }^{3}$ Department of Statistics, Regina Elena National Cancer Institute - IRCCS, Rome, ${ }^{4}$ Medical Oncology Unit 1, Department of Clinical and Experimental Oncology, Istituto Oncologico Veneto IOV - IRCCS, Padova, ${ }^{5}$ Department of Medical Oncology, Azienda Ospedaliero-Universitaria Pisana Spedali Riuniti S. Chiara, Pisa, ${ }^{6}$ Department of Medical Oncology, Policlinico Umberto I, Roma, ${ }^{7}$ Department of Medical Oncology, Azienda Ospedaliero-Universitaria di Santa Maria, Terni, ${ }^{8}$ Department of Uro-Gynaecological Oncology, Istituto Nazionale Tumori IRCCS "Fondazione G. Pascale", Napoli, ${ }^{9}$ Department of Oncology and Hematology, Azienda Ospedaliero-Universitaria Policlinico di Modena, Modena, ${ }^{10}$ Department of Medical Oncology, Istituto Nazionale Tumori - IRCCS, Aviano, ${ }^{17}$ Medical Oncology Unit, Azienda Ospedaliero-Universitaria di Parma, Parma, ${ }^{12}$ Department of Medical Oncology, CRO Pordenone-S. Vito Oncology, CRO - IRCCS, Aviano, ${ }^{73}$ Department of Medical Oncology, ASST Spedali Civili di Brescia, Brescia, ${ }^{14}$ Department of Medical Oncology, Azienda USL 8, Arezzo, ${ }^{15}$ Department of Medical Oncology, Regina Elena National Cancer Institute - IRCCS, Rome, ${ }^{16}$ Department of Medical Oncology, Fermo Area Vasta 4, Fermo, ${ }^{17}$ Department of Medical Oncology, Azienda Ospedaliero-Universitaria San Martino IST - IRCCS, Genova, ${ }^{18}$ University of Pavia and IRCCS San Matteo University Hospital Foundation, Pavia, ${ }^{19}$ IRCCS San Matteo University Hospital Foundation, Pavia, ${ }^{20}$ Department of Medical Oncology, Ospedale Santa Maria delle Croci, Ravenna, ${ }^{27}$ Department of Medical Oncology, San Camillo Forlanini Hospital, Roma, and ${ }^{22}$ Department of Medical Oncology, Istituto Nazionale Tumori - IRCCS, Milano, Italy

Note: These data were presented in part during a poster session at the 2017 American Society of Clinical Oncology Annual Meeting (Abstract \# 4577).

C.N.S. and G.P. are co-senior authors

aDetails of the Italian Nivolumab Renal Cell Cancer Early Access Programme Group are given in the Acknowledgements section.
}

\section{Objective}

To report the safety and efficacy results of patients enrolled in the Italian Nivolumab Renal Cell Cancer Expanded Access Programme.

\section{Patients and Methods}

Patients with metastatic renal cell cancer (mRCC) previously treated with agents targeting the vascular endothelial growth factor pathway were eligible to receive nivolumab $3 \mathrm{mg} / \mathrm{kg}$ once every 2 weeks. Patients included in the analysis had received $\geq 1$ dose of nivolumab and were monitored for adverse events (AEs) using Common Terminology Criteria for Adverse Events (CTCAE) v.4.0.

\section{Results}

A total of 389 patients were enrolled between July 2015 and April 2016, of whom $18 \%$ were aged $\geq 75$ years, $6.7 \%$ had non-clear cell RCC, $49.6 \%$ had bone and $8.2 \%$ brain metastases, and $79 \%$ had received $\geq 2$ previous lines of therapy. The most common any-grade treatment-related AEs were fatigue (13\%) and rash (9\%). Twenty-two patients (5.7\%) discontinued treatment because of AEs. There were no treatment-related deaths. The objective response rate was $23.1 \%$. At a median follow-up of 12 months, the median progression-free survival was 4.5 months (95\% confidence interval 3.7-6.2) and the 12-month overall survival rate was $63 \%$. Similar survival rates were reported among patients with non-clear-cell histology, elderly patients, those with bone and/ or brain metastases, and those who had received prior firstline sunitinib or pazopanib, or prior everolimus.

\section{Conclusion}

The safety and efficacy observed were consistent with those reported in the pivotal Checkmate 025 trial. Results in 
patients with non-clear-cell mRCC who were elderly, pretreated with everolimus, and had bone and/or brain metastases encourage the use of nivolumab in these categories of patients.

\section{Keywords}

renal cell cancer, nivolumab, expanded access programme, real-world experience

\section{Introduction}

Nivolumab is a fully human programmed death (PD)-1) immune checkpoint inhibitor antibody, which blocks the interaction between PD-1 expressed on T cells and its ligands PD-L1 and PD-L2, expressed on antigen-presenting cells and cancer cells [1]. Nivolumab therapy induces disruption of PD-1-PD-L1 signalling, restoring the ability of T cells to selectively recognize and kill cancer cells [2].

In a randomized phase III trial (CheckMate 025) in patients with metastatic RCC (mRCC), nivolumab administered after previous vascular endothelial growth factor (VEGF)-targeted therapy improved the median overall survival (OS) by 5.4 months and had a more favourable safety profile compared with everolimus [3]. This difference was both statistically significant as well as clinically relevant. In November 2015, the US Food and Drug Administration, and in February 2016 the European Medicines Agency, approved nivolumab for patients with $\mathrm{mRCC}$ who have received prior anti-angiogenic therapy [4,5]. A major concern, however, is whether patients enrolled in clinical trials are representative of the overall $\mathrm{mRCC}$ patient population. A large number of patients with mRCC do not meet criteria for enrolment in phase III registrative trials, such as those with poor performance status and those with brain metastases, who represent nearly $10 \%$ and $15 \%$ of patients with mRCC, respectively, after one or more lines of treatment [6,7]. Additionally, mRCC trials exclude cases with non-clear-cell histologies which represent $15-20 \%$ of cases [8,9]; thus, little is known about the activity of new agents in these categories of patient.

The Italian Nivolumab Renal Cell Cancer Expanded Access Programme (EAP) was initiated in July 2015, based on the key clinical data described above, while nivolumab was evaluated by the European Medicines Agency and negotiations with the Italian Ministry of Health were ongoing, to address the unmet medical need for patients whose disease progressed after receiving VEGF-targeted therapy.

In the present study, we report the results of patients enrolled in the Italian EAP and seek to evaluate the safety and efficacy of nivolumab in mRCC in a real-world setting.

\section{Patients and Methods}

\section{Study Population}

Between July 2015 and April 2016 nivolumab was provided by Bristol-Myers Squibb through the EAP to 95 hospitals in
Italy. A total of 490 requests were authorized, but 389 patients $(80 \%)$ ultimately received at least one dose of nivolumab, while most of the remaining $20 \%$ of patients experienced further disease progression with clinical deterioration prior to treatment.

Eligible patients were aged $\geq 18$ years and had mRCC that had relapsed after at least one prior anti-angiogenic therapy regimen (including, but not limited to, sunitinib, sorafenib, pazopanib, axitinib, tivozanib and bevacizumab). Prior mammalian target of rapamycin (mTOR) inhibitors and cytokine therapy (e.g. interleukin-2, interferon), vaccine therapy, or treatment with cytotoxic agents were also permitted. There was no limitation on the number of prior treatment regimens allowed. Patients were divided into three risk categories: favourable, intermediate and poor, according to the International Metastatic Renal Cell Carcinoma Database Consortium (IMDC) [10]. Inclusion criteria permitted the presence of asymptomatic brain metastases allowing systemic treatment with corticosteroids equivalent to up to $10 \mathrm{mg}$ daily prednisone. Mildly impaired renal function was allowed, including serum creatinine $\leq 1.5 \times$ the upper limit of normal or creatinine clearance $\geq 40 \mathrm{~mL} / \mathrm{min}$. Patients with active autoimmune disease were excluded. Patients with non-clear-cell histologies were also included in the present study through an amendment during the last 3 months of accrual. The study protocol was reviewed and approved by ethics committees. Patients signed and dated a written informed consent form provided by Bristol-Myers Squibb. All data presented were prospectively collected on electronic patient files.

\section{Treatment}

Nivolumab $3 \mathrm{mg} / \mathrm{kg}$ was administered intravenously every 2 weeks until withdrawal of consent, unacceptable toxicity or disease progression, at the discretion of the physician.

Safety assessments included physical evaluation and laboratory examination the day before every nivolumab administration. Blood tests included haematology, renal and hepatic function, pancreatic enzymes and hormone levels (thyroid function, adrenocorticotropic hormone, cortisol). Data on treatment-related adverse events (AEs), and especially immuno-related AEs, as reported by each treating physician were obtained from patient clinical files and laboratory reports, and classified according to the Common Terminology Criteria for Adverse Events v4.0. Treatment beyond Response Evaluation Criteria in Solid Tumors 
(RECIST) v1.1-defined progression was allowed in patients deriving investigator-assessed clinical benefit in the absence of rapid disease progression and tolerating the immunological treatment. A radiographic CT assessment was performed every 3 months and within 6 weeks of original progressive disease to confirm whether there was a decrease or stability in the tumour size or continued progressive disease.

\section{Statistical Analysis}

Data were summarized as frequencies for categorical variables and median and range values for continuous variables.

Continuous variables were compared using the Wilcoxon test. Associations between categorical variables were assessed using Fisher's exact test, when appropriate. Differences were considered statistically significant when $P<0.05$. Progressionfree survival (PFS) was calculated from the start of nivolumab treatment until disease progression or death. Alive patients without progression were censored at the time of last follow-up. OS was calculated from the start of nivolumab treatment until death. Patients lost to follow-up were censored at the time of last contact. The Kaplan-Meier method was used to estimate PFS and OS. The log-rank test and Cox proportional hazards regression were used to test for differences between groups. After univariate analysis, a multivariate analysis was carried out using a Cox regression model. All statistical analyses were performed by an experienced biostatistician with SPSS Statistical Software, version 21.0 (IBM_SPSS, Armonk, NY, USA).

\section{Results}

\section{Patients}

This analysis included all 389 patients who were enrolled in the EAP at 95 centres in Italy and were treated with $\geq 1$ dose of nivolumab, with a median (range) follow-up of 11.9 (124.7) months. Baseline patient characteristics are shown in Table 1. Patients received a median (range) of 13 (1-49) doses of nivolumab. At the time of the analysis 110 patients (28.3\%) were continuing treatment; among the 279 patients (71.7\%) who discontinued treatment, the reasons for discontinuation were progressive disease in 213 patients (76.3\%), death in $21(7.5 \%)$ and serious AEs in $22(7.9 \%)$.

\section{Safety}

Treatment-related AEs are shown in Table 2. Treatmentrelated grade 3-4 AEs occurred in 27 patients (7\%). Of the 22 serious AEs that induced treatment discontinuation, 11 (50\%) were considered potentially immuno-related AEs including: grade 4 hyperglycaemia with grade 3 diarrhoea $(n=1)$; grade 3 pneumonitis $(n=1)$; grade 3 bronchiolitis obliterans organizing pneumonia, grade 3 asthenia $(n=1$,) grade 3 hypertension $(n=1)$; grade 3 skin toxicity $(n=1)$; grade 3
Table 1 Patient characteristics $(N=389)$.

\begin{tabular}{|c|c|}
\hline Characteristics & \\
\hline Men, $n(\%)$ & $291(74.8)$ \\
\hline Median (range) age, years & $65(34-85)$ \\
\hline Age $\geq 75$ years, $n(\%)$ & $70(18.0)$ \\
\hline \multicolumn{2}{|c|}{ ECOG performance status, $n(\%)$} \\
\hline 0 & $176(47.1)$ \\
\hline 1 & $174(46.5)$ \\
\hline 2 & $24(6.4)$ \\
\hline NA & 15 \\
\hline \multicolumn{2}{|l|}{ IMDC prognostic group, $n(\%)$} \\
\hline Favourable & $62(20.2)$ \\
\hline Intermediate & $212(69.1)$ \\
\hline Poor & $33(10.7)$ \\
\hline NA & 82 \\
\hline Nephrectomy, $n$ (\%) & $369(94.9)$ \\
\hline \multicolumn{2}{|l|}{ Histology, $n(\%)$} \\
\hline Clear-cell & $356(91.5)$ \\
\hline Non-clear-cell & $26(6.7)$ \\
\hline Undifferentiated/Unknown & $7(1.8)$ \\
\hline \multicolumn{2}{|l|}{ Metastasis site, $n(\%)$} \\
\hline Lung & $286(73.5)$ \\
\hline Lymph node & $238(69.2)$ \\
\hline Bone & $193(49.6)$ \\
\hline Liver & $128(32.9)$ \\
\hline Brain & $32(8.2)$ \\
\hline \multicolumn{2}{|c|}{ Number of prior systemic therapies, $n(\%)$} \\
\hline 1 & $80(20.7)$ \\
\hline 2 & $137(35.4)$ \\
\hline$\geq 3$ & $170(43.9)$ \\
\hline \multicolumn{2}{|l|}{ First-line therapy, $n(\%)$} \\
\hline Sunitinib & $261(67.4)$ \\
\hline Pazopanib & $80(20.7)$ \\
\hline Other & $46(11.9)$ \\
\hline \multicolumn{2}{|l|}{ Prior everolimus, $n(\%)$} \\
\hline Yes & $163(42.1)$ \\
\hline Not & $224(57.9)$ \\
\hline
\end{tabular}

tremor $(n=1)$; grade 2 eyelid ptosis $(n=2)$; grade 2 liver toxicity $(n=1)$; and grade 2 hypothyroidism $(n=1)$. AEs were generally manageable with treatment as per protocolspecific guidelines. No treatment-related deaths were reported.

\section{Tumour Assessment}

Response evaluations were available for 355 (91.3\%) of 389 included patients, whereas the remaining 34 cases were not assessable as a result of early death $(n=22)$, early discontinuation attributable to toxicity after a median of 3 cycles $(n=5)$, loss to follow-up $(n=4)$, and unspecified reasons $(n=3)$. The best overall response in the overall patient population was complete response in three patients $(0.8 \%)$, partial response in $87(22.4 \%)$, stable disease in 124 (31.9\%) and progressive disease in 141 (36.2\%); thus, the objective response rate (ORR) was $23.1 \%$ (90/389) in the overall population and $25.4 \%(90 / 355)$ in patients who had $\geq 1$ response assessment reported. Response rates among patients were irrespective of age, histology, previous lines of 
Table 2 Rates of adverse events reported in the CheckMate 025 trial and in the Italian Expanded Access Programme.

\begin{tabular}{|c|c|c|c|c|c|c|}
\hline & \multicolumn{4}{|c|}{ CheckMate 025 [3] } & \multirow{2}{*}{\multicolumn{2}{|c|}{$\begin{array}{c}\text { Italian EAP } \\
\begin{array}{c}\text { Nivolumab } \\
N=389\end{array}\end{array}$}} \\
\hline & \multicolumn{2}{|c|}{$\begin{array}{l}\text { Everolimus } \\
\qquad N=397\end{array}$} & \multicolumn{2}{|c|}{$\begin{array}{c}\text { Nivolumab } \\
\qquad \begin{array}{l}N=6.06\end{array}\end{array}$} & & \\
\hline & Any grade & Grade $\geq 3$ & Any grade & Grade $\geq 3$ & Any grade & Grade $\geq 3$ \\
\hline Treatment-related AEs, \% & 88 & 37 & 79 & 19 & 32 & 7 \\
\hline Fatigue & 34 & 3 & 33 & 2 & 13 & 2 \\
\hline Pyrexia & NR & NR & NR & NR & 3 & 0 \\
\hline Nausea & 17 & 1 & 14 & $<1$ & 0 & 0 \\
\hline Pruritus & 10 & 0 & 14 & 0 & 0 & 0 \\
\hline Diarrhoea & 21 & 1 & 12 & 1 & 5 & 1 \\
\hline Decreased appetite & 21 & 1 & 12 & $<1$ & 1 & $<1$ \\
\hline Rash & 20 & 1 & 10 & $<1$ & 9 & $<1$ \\
\hline Hypothyroidism & NR & NR & NR & NR & 2 & 0 \\
\hline Hyperthyroidism & NR & NR & NR & NR & 2 & 0 \\
\hline Hypophysitis & NR & NR & NR & NR & $<1$ & $<1$ \\
\hline Hypertransaminases & NR & NR & NR & NR & 1 & 0 \\
\hline Cough & 19 & 0 & 9 & 0 & 0 & 0 \\
\hline Anaemia & 24 & 8 & 8 & 2 & 2 & $<1$ \\
\hline Dyspnoea & 13 & $<1$ & 7 & 1 & 3 & 1 \\
\hline Oedema peripheral & 14 & $<1$ & 4 & 0 & 0 & 0 \\
\hline Pneumonitis & 15 & 3 & 4 & 1 & 2 & $<1$ \\
\hline Mucosal inflammation & 19 & 3 & 3 & 0 & 0 & 0 \\
\hline Dysgeusia & 13 & 0 & 3 & 0 & 0 & 0 \\
\hline Hyperglycaemia & 12 & 3 & 2 & 1 & 0 & 0 \\
\hline Stomatitis & 29 & 4 & 2 & 0 & 0 & 0 \\
\hline Hypertriglyceridaemia & 16 & 4 & 1 & 0 & 0 & 0 \\
\hline Epistaxis & 10 & 0 & 1 & 0 & 0 & 0 \\
\hline
\end{tabular}

$A E$, adverse event; EAP, Nivolumab RCC Expanded Access Programme; NR, not reported.

therapy, brain and bone metastasis (Table 3). A total of 107 patients $(27.5 \%)$ were treated beyond progression. Among these latter patients, a subsequent response or stabilization was achieved in 40 patients (37.4\%), including partial response in $10(9.3 \%)$ and stable disease in $30(28 \%)$, whereas progressive disease was reported in 62 patients (57.9\%), and response was not assessable in five patients (4.7\%). The 12month OS rate of these 107 patients was $77.4 \%$ (95\% CI 69.0-85.8).

\section{Survival}

The 6-, 12- and 18-month OS rates were $80.0 \%$ (95\% CI 75.9-84.1), 63.1\% (95\% CI 58.2-68.0), and 53.8\% (95\% CI 48.3-59.3), respectively (Fig. 1A). At the time of the analysis, the median OS was not yet reached. The median PFS was 4.4 months (95\% CI 3.7-6.2; Fig. 1B). The median time on therapy was 7.2 months (95\% CI 6.1-8.3).

In univariate analysis, age, performance status, IMDC prognostic group and number of prior therapies were found to be significantly associated with OS (Table 4). Prior firstline treatment with sunitinib or pazopanib did not show any correlation with OS after nivolumab treatment either in the global series (Figure S1A) or in cases with second-line nivolumab treatment (Figure S1B), whereas prior everolimus showed a borderline association with OS (Table 4).
In multivariate analysis, age, IMDC score and number of previous lines of treatment all remained predictors of OS $(P=0.04, P<0.0001$ and $P=0.02$, respectively; Table 4$)$.

\section{Discussion}

The treatment of patients with $\mathrm{mRCC}$ has undergone great progress in the last 10 years with the arrival of a number of active agents, including, more recently, the immune checkpoint inhibitor nivolumab $[3,11,12]$. These drugs provide new opportunities, but the implication for general practice is largely undocumented [11]. Moreover, patient populations in phase II or III trials may differ from those experienced by physicians in clinical practice [13]. This analysis evaluated the safety and efficacy of nivolumab in patients with mRCC treated in the EAP in Italy.

In this real-life analysis, nivolumab showed satisfactory safety and tolerability, with only $7.9 \%$ of patients discontinuing treatment because of serious AEs. We focused on treatmentrelated toxicities and found lower incidences of grade 3-4 AEs (7\%) than reported in the CheckMate 025 trial (19\%; Table 2) [3]. This may be partially explained by a slight underreporting of side effects as patients were treated or even admitted in local hospitals, and these events may not be regularly registered; however, the broad mRCC population in the Italian EAP included $49.6 \%$ of patients with bone and/or $8.2 \%$ with brain metastases, $79.3 \%$ treated from the third-line 
Table 3 Best response in subpopulations of patients.

\begin{tabular}{lc}
\hline Population/best response & $n(\%)$ \\
\hline Overall population $(N=389)$ & \\
Complete response & $3(0.8)$ \\
Partial response & $87(22.4)$ \\
Stable disease & $124(31.9)$ \\
Progressive disease & $141(36.2)$ \\
Could not be determined & $34(8.7)$ \\
Age $\geq 75$ years $(n=70)$ & \\
Complete response & $1(1.6)$ \\
Partial response & $19(30.2)$ \\
Stable disease & $24(38.1)$ \\
Progressive disease & $19(30.2)$ \\
Could not be determined & $7(8.8)$ \\
Non-clear-cell histology $(N=26)$ & \\
Complete response & 0 \\
Partial response & $5(19.2)$ \\
Stable disease & $3(11.5)$ \\
Progressive disease & $14(53.8)$ \\
Could not be determined & $4(15.4)$ \\
Brain metastasis $(n=32)$ & \\
Complete response & $1(3.1)$ \\
Partial response & $5(15.6)$ \\
Stable disease & $11(34.4)$ \\
Progressive disease & $13(40.6)$ \\
Could not be determined & $2(6.2)$ \\
Bone metastasis $(n=193)$ & \\
Complete response & $1(0.5)$ \\
Partial response & $37(19.2)$ \\
Stable disease & $57(29.5)$ \\
Progressive disease & $86(44.6)$ \\
Could not be determined & $12(6.2)$ \\
Patients treated $\geq$ third line $(N=307)$ & \\
Complete response & $2(0.7)$ \\
Partial response & $60(19.5)$ \\
Stable disease & $98(31.9)$ \\
Progressive disease & $120(39.1)$ \\
Could not be determined & $27(8.8)$ \\
\hline & \\
\hline & \\
\hline
\end{tabular}

setting and $10.7 \%$ poor-risk patients (Table 1 ). These are all subgroups that might be predicted to tolerate therapy less well than patients in the selected population in the phase III trial, which included only $19 \%$ of bone metastases and $16 \%$ of patients deemed poor risk. In the registration study, patients with brain metastases and those treated in a fourthline setting were not included [3]. Despite this less-defined patient population with poorer prognosis, in the EAP analysis efficacy results of nivolumab for the treatment of mRCC were similar to those of the CheckMate 025 trial [3]. In particular, the ORRs in the EAP and in Checkmate 025 were $23.1 \%$ and $25 \%$, respectively; the median PFS was 4.4 and 4.6 months, respectively; median OS was not reached in the EAP; however, the 12 -month OS was $63 \%$, which approached the $76 \%$ observed in the nivolumab arm of the CheckMate 025 trial. Subgroup analyses showed that the ORR did not differ among the following groups: elderly patients; those with nonclear-cell histologies; different numbers of prior treatment lines; or patients with bone and/or brain metastases (Table 3). In particular, the cohort of 32 patients with brain metastases was not associated with differences compared with the overall
Fig. 1 Kaplan-Meier estimate of (A) overall survival and (B) progressionfree survival in all patients.
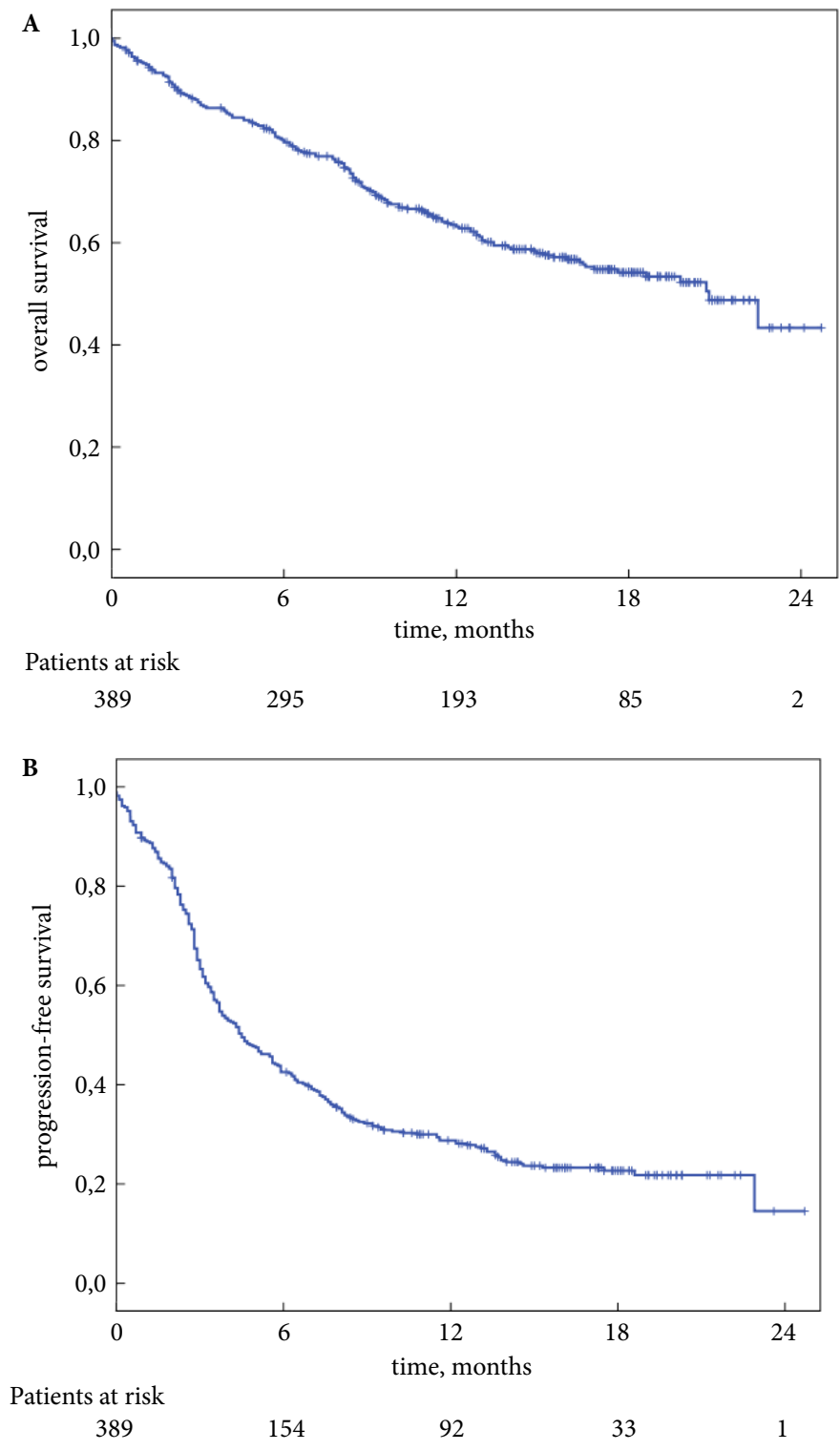

population in terms of ORR (Table 3), and no association with OS was observed after univariate analysis (Table 4); this effect could be explained by cerebral activity of nivolumab as suggested by other authors [14]. Data from the IMDC in patients with mRCC treated with second-line VEGF-targeted therapy showed that patients with non-clear-cell mRCC had a worse ORR than their counterparts with clear-cell RCC (8\% vs $12 \%$, respectively) [15]. The 26 cases with $\mathrm{mRCC}$ with non-clear-cell histology in our series showed a slightly inferior response rate of $19.2 \%$ vs $23.1 \%$ of the overall population, suggesting that nivolumab is an active agent for these tumours, even if longer follow-up is needed to obtain mature data on OS. Other authors have recently reported an 
Table 4 Association between baseline characteristics and overall survival.

\begin{tabular}{|c|c|c|c|c|}
\hline \multirow[t]{2}{*}{ Characteristic } & \multicolumn{2}{|c|}{ Univariate analysis } & \multicolumn{2}{|c|}{ Multivariate anclysis } \\
\hline & HR $(95 \%$ Cl) & $\boldsymbol{P}$ & HR $(95 \%$ Cl) & $\boldsymbol{P}$ \\
\hline \multicolumn{5}{|l|}{ Age } \\
\hline$\geq 75$ vs $<75$ years & $0.62(0.39-0.98)$ & 0.04 & $0.55(0.32-0.96)$ & 0.04 \\
\hline \multicolumn{5}{|l|}{ Gender } \\
\hline Male vs female & $1.18(0.82-1.71)$ & 0.36 & - & \\
\hline \multicolumn{5}{|l|}{ Metastatic site, yes vs no } \\
\hline Bone & $1.35(0.99-1.84)$ & 0.06 & - & \\
\hline Liver & $1.05(0.76-1.46)$ & 0.75 & - & \\
\hline CNS & $1.39(0.84-2.31)$ & 0.20 & - & \\
\hline \multicolumn{5}{|l|}{ Number of prior therapies } \\
\hline$>1$ vs 1 & $1.80(1.15-2.87)$ & 0.01 & $1.87(1.13-3.09)$ & 0.01 \\
\hline \multicolumn{5}{|l|}{ IMDC prognostic group } \\
\hline Intermediate vs favourable & $1.97(1.18-3.31)$ & 0.01 & $2.06(1.23-3.46)$ & 0.006 \\
\hline Poor vs favourable & $4.20(2.23-7.93)$ & $<0.0001$ & $4.34(2.30-8.20)$ & $<0.001$ \\
\hline \multicolumn{5}{|l|}{ First-line treatment } \\
\hline Sunitinib vs pazopanib & $1.24(0.84-1.84)$ & 0.28 & & \\
\hline \multicolumn{5}{|l|}{ Prior everolimus } \\
\hline Yes vs no & $1.30(0.95-1.76)$ & 0.10 & & \\
\hline
\end{tabular}

HR, hazard ratio; IMDC, International Metastatic Renal Cell Carcinoma Database Consortium.

objective response in seven (20\%) of 35 patients with nonclear-cell mRCC treated with nivolumab, but even in that study follow-up was too short to have data on OS [16]. Larger series including different histological subtypes with longer follow-up are needed to better characterize the biological activity of nivolumab in these subsets of tumours.

In a post hoc analysis of the Checkmate 025 study, the impact of baseline characteristics, including prognostic score and prior therapy, on the clinical outcome has been recently evaluated [17]. The median OS was longer in both arms in patients with better IMDC prognostic scores and with prior sunitinib or pazopanib therapy. Moreover, a trend towards better OS for patients treated with nivolumab with prior pazopanib vs sunitinib was observed in the Kaplan-Meier analyses [17]; however, an interaction test revealed a significant interaction for risk group only but no evidence for other subgroups. In line with these results, in the Italian EAP, IMDC score was the strongest factor able to predict OS, whereas no impact of prior sunitinib or pazopanib was shown (Table 4 and Figure S1A,B).

In the preliminary exploratory analysis of the French Nivoren study of 528 consecutive patients with mRCC treated with nivolumab as a second or further line of treatment, the authors showed a borderline but statistically significant prognostic impact of previous everolimus (hazard ratio [HR] 1.39, CI 1.01-1.92; $P=0.044)$, whereas there was no impact of the number of the prior lines measured as $>2$ vs $\leq 2$ (HR 1.05, CI 0.75-1.48; $P=0.764$ ) [18]. In the Italian EAP, everolimus showed just a trend for inferior OS (HR 1.30, CI $0.95-1.76 ; P=0.10$ ), whereas the number of prior lines measured as $>1$ vs $\leq 1$ showed a statistically significant impact on OS (HR 1.80, CI 1.15-2.87; $P=0.01$ ) that was confirmed in multivariate analysis (Table 4). Until recently, everolimus has been used as standard of care as a second or further line of therapy. Patients treated with one prior line only did not receive everolimus; therefore, patients treated with nivolumab after everolimus could have a prognostic impact because of more prior lines of therapy received.

Despite the efficacy of nivolumab, a relevant unmet need remains the development of immuno-inflammatory biomarkers able to predict long-term clinical benefit in order to identify which patients with mRCC are likely to have an advantage from this therapy [19-22]. This is extremely relevant for the management of nivolumab in patients with mRCC with an ORR of $25 \%$, and with long-term survival $>4$ years in nearly $20 \%$ of cases $[10,11]$.

This EAP provides extensive real-world experience with nivolumab in patients with previously treated mRCC, including a consistent number of elderly patients and patients with bone metastases, who were poorly represented in the pivotal CheckMate 025 study, and patients with brain metastases and non-clear-cell histotypes, who were not represented at all [3]. Despite these differences, the safety profile and efficacy of nivolumab appeared consistent with that reported in the pivotal trial [3]. Noting the limitations of this type of study, preliminary data from this EAP appear to confirm data from the pivotal trial and suggest that nivolumab is safe and efficacious for the treatment of mRCC in routine clinical practice.

\section{Acknowledgements}

This data collection was financially supported by BristolMyers Squibb. The financial sponsor of the trial had no 
role in the design or conduct of the trial, data collection or analysis and preparation of the manuscript. The authors wish to thank all the patients and their families who participated in the Italian RCC EAP as well as all the investigators: U. De Giorgi (Meldola), G. Procopio (Milan), G. Cartenì (Naples), A. Falcone (Pisa), U. Basso (Padova), E. Cortesi (Rome); F. Roila (Terni), S. Cascinu (Modena), U. Tirelli (Aviano), S. Buti (Parma), S. Pignata (Naples), G. Lo Re (Pordenone), A. Berruti (Brescia), S. Bracarda (Arezzo), F. Cognetti (Roma), L. Giustini (Fermo), A. Sobrero (Genova), D. Turci (Ravenna), C. N. Sternberg (Roma), C. Porta (Pavia), F. Cappuzzo (Livorno), G. Tortora (Verona), D. Tassinari (Rimini), R. Passalacqua (Cremona), A. Pazzola (Sassari), G. Surico (Lecce), M. Maio (Siena), G. Benedetti (Macerata), C. Barone (Roma); V. Adamo (Messina), E. Ricevuto (L’Aquila), A. De Censi (Genova), M. Spada (Cefalù), G. Tonini (Roma), C. Pinto (Reggio Emilia), L. Ciuffreda (Torino), E. M. Ruggeri (Viterbo), C. Bengala (Grosseto), V. Scotti (Florence), D. Fagnani (Vimercate), A. Bonetti (Legnago), M. Mitterer (Merano), F. Castiglione (Cuneo), P. Bidoli (Monza), F. Ferraù (Taormina), L. Crinò (Perugia), A. Frassoldati (Ferrara), P. Marchetti (Roma), E. Mini (Florence), A. Scoppola (Roma), C. Verusio (Saronno), A. Favaretto (Treviso), F. Di Costanzo (Florence), G. Fasola (Udine), M. Merlano (Cuneo), F. Artioli (Carpi), A. Di Leo (Prato), S. Romito (Foggia), A. Maestri (Imola), C. Giannitto Giorgio (Caltagirone), M. T. Ionta (Cagliari), F. Verderame (Palermo), G. Zampa (Roma), G. Numico (Alessandria), M. Minelli (Roma), P. Tagliaferri (Catanzaro), P. Foa (Milan), G. Palmiotti (Bari), S. De Placido (Naples), R. Mattioli (Fano), F. Iuliano (Rossano), E. Defraia (Cagliari), S. Siena (Milano), M. Clerico (Biella), L. Salvagno (Vittorio Veneto), G. L. Ceresoli (Bergamo), A. Bernardo (Pavia), M. Di Lieto (Pistoia), M. Moroni (Milan), M. Maisano (Reggio Calabria), M. Scartozzi (Cagliari), G. Scagliotti (Orbassano), M. Sorarù (Cittadella), S. Pepe (Salerno), A. Scaltriti (Guastalla), V. Gebbia (Palermo), E. Testa (Urbino), V. Lorusso (Bari), R. Bordonaro (Catania), G. De Signoribus (S. Benedetto del Tronto), N. Tedde (Olbia), A. Santoro (Rozzano), G. Francini (Siena), G. Aondio (Gravedona).

\section{Conflict of Interest}

Ugo De Giorgi plays a consultant/advisory role in BristolMyers Squibb, Ipsen, Pfizer, Novartis, Astellas, Janssen, Sanofi. Umberto Basso received speaker fees from BristolMyers Squibb, Pfizer, Novartis, Pierre Fabre, Janssen, Astellas, Sanofi, and has had an advisory role for Sanofi, Novartis, MSD, Janssen. Roberto Sabbatini plays a consultant/advisory role in Bristol-Myers Squibb, Ipsen, Pfizer, Novartis, Astellas, Janssen, Sanofi. Cora N. Sternberg received honoraria from Bristol-Myers Squibb, Novartis, Pfizer, Ipsen, Eisai.

\section{References}

1 Topalian SL, Hodi FS, Brahmer JR et al. Safety, activity, and immune correlates of anti-PD-1 antibody in cancer. $N$ Engl J Med 2012; 366: 2443-54

2 Farolfi A, Schepisi G, Conteduca V, Burgio SL, Lolli C, De Giorgi U. Pharmacokinetics, pharmacodynamics and clinical efficacy of nivolumab in the treatment of metastatic renal cell carcinoma. Expert Opin Drug Metab Toxicol 2016; 12: 1089-96

3 Motzer RJ, Escudier B, McDermott DF et al. Nivolumab versus everolimus in advanced renal-cell carcinoma. N Engl J Med 2015; 373: 1803-13

4 Administration USFD. FDA Expands Use of Immunotherapeutic to Kidney Cancer (2015). Available at: https://blog.aacr.org/fda-approvalnivolumab-kidney-cancer/. Accessed July 2018

5 Agency EM. New treatment for advanced form of kidney cancer (2016). Available at: http://www.ema.europa.eu/docs/en_GB/document_library/Press_ release/2016/02/WC500202416.pdf. Accessed July 2018

6 Vickers MM, Al-Harbi H, Choueiri TK et al. Prognostic factors of survival for patients with metastatic renal cell carcinoma with brain metastases treated with targeted therapy: results from the international metastatic renal cell carcinoma database consortium. Clin Genitourin Cancer 2013; 11: 311-5

7 Gore ME, Szczylik C, Porta C et al. Safety and efficacy of sunitinib for metastatic renal-cell carcinoma: an expanded-access trial. Lancet Oncol 2009; 10: 757-63

8 Sankin A, Hakimi AA, Hsieh JJ, Molina AM. Metastatic non-clear cell renal cell carcinoma: an evidence based review of current treatment strategies. Front Oncol 2015; 5: 67

9 de Velasco G, McKay RR, Lin X, Moreira RB, Simantov R, Choueiri TK. Comprehensive analysis of survival outcomes in non-clear cell renal cell carcinoma patients treated in clinical trials. Clin Genitourin Cancer 2017; 15: 652-60

10 Ko JJ, Xie W, Kroeger $\mathrm{N}$ et al. The International Metastatic Renal Cell Carcinoma Database Consortium model as a prognostic tool in patients with metastatic renal cell carcinoma previously treated with first-line targeted therapy: a population-based study. Lancet Oncol 2015; 16: 293-300

11 Motzer RJ, Rini BI, McDermott DF et al. Nivolumab for metastatic renal cell carcinoma: results of a randomized phase II trial. J Clin Oncol 2015; 33: $1430-7$

12 McDermott DF, Drake CG, Sznol M et al. Survival, durable response, and long-term safety in patients with previously treated advanced renal cell carcinoma receiving nivolumab. J Clin Oncol 2015; 33: 2013-20

13 Joseph RW, Chatta G, Vaishampayan U. Nivolumab treatment for advanced renal cell carcinoma: considerations for clinical practice. Urol Oncol 2017; 35: 142-8

14 Dudnik E, Yust-Katz S, Nechushtan $\mathrm{H}$ et al. Intracranial response to nivolumab in NSCLC patients with untreated or progressing CNS metastases. Lung Cancer 2016; 98: 114-7

15 Kroeger N, Xie W, Lee JL et al. Metastatic non-clear cell renal cell carcinoma treated with targeted therapy agents: characterization of survival outcome and application of the International mRCC database consortium criteria. Cancer 2013; 119: 2999-3006

16 Koshkin VS, Barata PC, Zhang T et al. Clinical activity of nivolumab in patients with non-clear cell renal cell carcinoma. J Immunother Cancer 2018; 6: 9

17 Escudier B, Sharma P, McDermott DF et al. CheckMate 025 randomized phase 3 study: outcomes by key baseline factors and prior therapy for nivolumab versus everolimus in advanced renal cell carcinoma. Eur Urol 2017; 72: 962-71

18 Albiges L, Negrier S, Dalban C et al. Safety and efficacy of nivolumab in metastatic renal cell carcinoma (mRCC): Results from the NIVOREN GETUG-AFU 26 study. J Clin Oncol 2018; 36: 577 (suppl 6S; abstr 577) 
19 Pal SK, Sonpavde G, Agarwal N et al. Evolution of circulating tumor DNA profile from first-line to subsequent therapy in metastatic renal cell carcinoma. Eur Urol 2017; 72: 557-64

20 Lolli C, Basso U, Derosa L et al. Systemic immune-inflammation index predicts the clinical outcome in patients with metastatic renal cell cancer treated with sunitinib. Oncotarget 2016; 7: 54564-71

21 Zhu J, Armstrong AJ, Friedlander TW et al. Biomarkers of immunotherapy in urothelial and renal cell carcinoma: PD-L1, tumor mutational burden, and beyond. J Immunother Cancer 2018; 6: 4

22 Miao D, Margolis CA, Gao W et al. Genomic correlates of response to immune checkpoint therapies in clear cell renal cell carcinoma. Science 2018; 359: 801-6

Correspondence: Ugo De Giorgi, MD, PhD, Department of Medical Oncology, Istituto Scientifico Romagnolo per lo Studio e la Cura dei Tumori (IRST) IRCCS, Via Maroncelli 40, I-47014 Meldola, Italy.

e-mail: ugo.degiorgi@irst.emr.it
Abbreviations: AE, adverse event; EAP, Nivolumab RCC Early Access Programme; IMDC, International Metastatic Renal Cell Carcinoma Database Consortium; mRCC, metastatic RCC; ORR, objective response rate; OS, overall survival; PD-1, programmed death-1; PFS, progression-free survival; VEGF, vascular endothelial growth factor.

\section{Supporting Information}

Additional supporting information may be found online in the Supporting Information section at the end of the article:

Figure S1. Kaplan-Meier estimate of overall survival (OS) of patients treated with first-line sunitinib vs pazopanib before nivolumab in every line of treatment (A) and treated with first-line sunitinib vs pazopanib before nivolumab in secondline treatment $(\mathrm{B})$. 